Marc Escrihuela-Villar*

\title{
A Note on the Equivalence of the Conjectural Variations Solution and the Coefficient of Cooperation
}

\begin{abstract}
This note shows that in a general symmetric quantity-setting oligopoly, the conjectural variations solution replicates that of a model where the "coefficient of cooperation" defined by Cyert and DeGroot (1973) is interpreted as the parameter indicating severity of competition.
\end{abstract}

Keywords: conjectural variations, oligopoly, relative profit JEL Classification: C72, L13, L41

\section{Introduction}

The conjectural variations oligopoly solution, first introduced by Bowley (1924), has been used in both applied theoretic and empirical industrial organization in order to obtain the entire range of outputs between the competitive and the monopolistic. In short, the idea is that a firm in an oligopolistic market believes that the quantity chosen will also affect the quantities chosen by rivals in such a way that each firm expects a one-unit change in its quantity to lead to a change of $1+\lambda$ in total output. By varying the value of $\lambda$ between -1 and $n-1$ (where $n$ is the number of firms), one obtains the whole range mentioned before. This model has often been criticized since ad hoc conjectural variations are generally inconsistent with rational behavior except at the equilibrium point (see, among others, Makowski 1987). It has also been argued that its solution is not entirely satisfactory from a game-theoretic point of view because it describes "dynamics" based on a static model. Basically, in the theoretical literature, conjectural variations have been criticized for their lack of theoretical

*Corresponding author: Marc Escrihuela-Villar, Departamento de Economa Aplicada, Universitat de les Illes Balears, Edificio Jovellanos Ctra, Valldemossa, km 7.5 07122, Palma de Mallorca, Baleares, Spain, E-mail: marc.escrihuela@uib.es 
foundations, at least in static models (for additional references and a discussion, see Martin (2002, 50-51)). In the same line Tirole (1988), for instance, states that in a static game, each firm's choice is independent of its rivals' choices and therefore firms cannot react to one another. Thus, any conjecture about one's opponents' reaction that differs from no reaction is irrational and this methodology is not theoretically satisfactory, as it does not subject itself to the discipline imposed by game theory. Few studies, though, have attempted to address this concern while remaining in a static framework by imposing the requirement that a firm's beliefs, or conjectures, be consistent (see among others Laitner 1980, Bresnahan 1981, Perry 1982 and Kamien and Schwartz 1983). ${ }^{1}$ Other authors have attempted to reconcile the dynamic oligopolistic competition models with the static conjectural variations equilibria. For instance, using the adjustment cost model, Dockner (1992) shows that a conjectural variations equilibrium can be interpreted as the outcome of a dynamic game. Cabral (1995) presents a similar result showing that, in linear oligopolies, there exists an exact correspondence between the conjectural variations solution and the solution of a quantity-setting repeated game with minimax punishments during $T$ periods. ${ }^{2}$

On the other hand, a different way of modeling the intensity of competition consists of assuming that each firm cares about its own profit plus a weighted average of the profits of the other firms (see, for instance, Symeonidis (2000) and (2008) for a duopoly or Matsumura et al. (2013) for an oligopoly). Then, using a payoff function based on relative performance, the intensity of competition can also be parametrized. Intermediate values of this parameter then might represent imperfect collusion and may be justified by reference to some implicit dynamic model of collusion, a reduced-form representation of which being the quantity competition subgame of this model. This formulation is closely related to the "coefficient of cooperation," defined by Cyert and DeGroot (1973) and also to the growing and more recent behavioral economics literature. Examples of this literature include Fehr and Schmidt (1999) that, based on evidence suggesting that cooperation motives are crucial, analyze a model where people decisions are also driven by fairness considerations. In the

1 The conjectural variations approach has also been used in the empirical industrial organization literature to estimate the degree of noncompetitiveness in an industry (see among others, Slade (1986) or Brander and Zhang (1990)).

2 Figuières et al. (2004) present an interesting discussion on how to interpret, in different economic situations, the static conjectural variations equilibrium as a reduced form for dynamic competition. An extensive survey to review the theoretical literature on conjectural variations equilibria and their variants is also provided. An anonymous referee is thanked for this reference. 
same spirit, Charness and Rabin (2002) design a range of simple experimental games to test that subjects are concerned with increasing social welfare and reciprocity. Indeed, several arguments have been used to justify the relative performance approach. For instance, the aforementioned approach is evolutionarily stable (see Vega-Redondo 1997). Furthermore, it can be assumed that sufficiently patient firms always achieve the highest level of collusion that is sustainable as an equilibrium of an infinitely repeated game given a number of parameters taken as exogenous at the competition stage. Therefore, under this interpretation, a fall in the importance given to rivals' profits might correspond to a lower critical discount factor in an infinitely repeated game. Two significant examples illustrate the trend towards the preference of this model instead of the conjectural variations approach. Symeonidis (2000, 58 footnote 4) states that this formulation "is free of some of the theoretical problems encountered in other approaches to modeling the intensity of short-run competition by way of a reduced-form parameter, such as the conjectural variations approach". ${ }^{3}$ In the same line, Matsumura et al. $(2013,543)$ state, “A classic approach to parameterizing competitiveness is to use the conjectural variation. However, this approach is criticized by many authors because it is not consistent with the Nash equilibrium. Our model provides an alternative approach that is consistent with game-theoretic rationality."

The present note shows, however, that in a general symmetric quantitysetting oligopoly both models are essentially equivalent. It is proved that, in a symmetric model, conjecturing some reaction of your rivals to a change in your output leads to the same equilibrium quantities as caring about the average profits of your rivals. This result proves useful to justify the use of the conjectural variations solution as a means of estimating the degree of oligopoly power as long as it represents precisely the essence of the widely accepted coefficient of cooperation that captures the idea of a payoff function based on relative performance. One advantage of the latter formulation is that the above-mentioned coefficient can also be interpreted as a degree of envy or altruism or as a situation in which each firm cares about the firm earning the highest profit among its rivals or each firm has one specific rival as a benchmark firm and cares about its profit only.

3 Equivalently,d'Aspremont and Ferreira (2009, 63) propose a representation of oligopolistic competition that is "a convenient tool to the theorist (it amounts to use a generalization of Cournot equilibrium), which is more game-theoretically founded than the conjectural variations theory." In fact, they use a "coefficient of collective concern" which is very similar to the coefficient of cooperation but adds some positive weight also to the total surplus. 


\section{Two Ways of Modeling the Intensity of Competition}

Assume an $n$-firm homogenous product oligopoly where $2 \leq n$, and $n$ is as large as we want but finite. The industry inverse demand function is given by the function $P(Q)$ where $Q=\sum_{i=1}^{n} q_{i}$ is the industry output and $q_{i}$ is the output of the $i$ th firm in the $n$ firm industry. The demand function is twice continuously differentiable and downward sloping. Firm $i, i=1, \ldots, n$, can produce according to a (common) cost function $C\left(q_{i}\right)$ that is increasing and continuously differentiable. In addition, the marginal cost function is assumed to be nondecreasing (namely, $C^{\prime \prime}\left(q_{i}\right) \geq 0$ ). ${ }^{4}$ The profit function of firm $i$, to be maximized by choice of $q_{i}$, is

$$
\Pi_{i}\left(q_{i}, Q_{-i}\right)=P(Q) q_{i}-C\left(q_{i}\right)
$$

In this baseline framework, two different ways of modeling the intensity of competition will be considered below.

Firstly, we take up conjectural variations (CV) and their properties. Let $\left(\partial Q_{-i} / \partial q_{i}\right) \equiv \lambda_{i}$ represent firm $i$ 's conjectural variation, or what is the same, its expectation regarding the change in its competitors production resulting from a change in its own production level with $Q_{-i} \equiv Q-q_{i}$ representing the quantity supplied by the $i$ th firm $(n-1)$ competitors. While conjectural variations can differ among firms we follow the simplifying assumption which is common in the literature (see, among others, Perry 1982 and Cabral 1995), that conjectures are identical and constant, i.e., $\lambda_{i}=\lambda$, for all $i$. Then, $\lambda$ is usually referred to as the competitive intensity of the industry, with lower values of $\lambda$ corresponding to more intense competition. Therefore, the industry equilibrium can vary from a competitive equilibrium to a perfectly collusive one as $\lambda$ moves from $\lambda=-1$ to $\lambda=n-1$.

On the other hand, a second attempt to allow for imperfect collusion involves assuming that each firm maximizes the sum of its own profit plus the average profits of the remaining firms in such a way that a generic firm $i$ maximizes

$$
\Pi_{i}\left(q_{i}, Q_{-i}\right)+\frac{\alpha}{n-1} \sum_{j \neq i}^{n} \Pi_{j}\left(q_{j}, Q_{-j}\right)
$$

4 Dixit (1986) obtains that $C^{\prime \prime}\left(q_{i}\right)-P^{\prime}(Q)>0$ is among the conditions for the stability of an equilibrium in a homogeneous product oligopoly. This condition is surely met if marginal cost is nondecreasing. 
where no conjecture about the reaction of the rivals is expected (namely, $\left.\left(\partial Q_{-i} / \partial q_{i}\right)=0\right)$. In this model, $\alpha$ it is assumed to be constant and symmetric and interpreted as the severity of competition in such a way that $\alpha=0$ indicates the standard Cournot case, $\alpha=-1$ the perfectly competitive case and $\alpha=n-1$ the monopoly case. Following Cyert and DeGroot (1973), we can denote this formulation by coefficient of cooperation model (CC). ${ }^{5}$

We concentrate on the symmetric equilibria of our symmetric oligopolies where the $n$ symmetric first-order conditions (FOCs) determine the equilibrium quantities in each model. In addition, the second-order conditions (SOCs) also need to be satisfied. In this sense, Vives (2001) shows that the (common) assumption of downward sloping marginal revenue $\left(\mathrm{MR}_{i}\right)$

$$
\frac{\partial \mathrm{MR}_{i}}{\partial Q_{-i}}=P^{\prime}(Q)+P^{\prime \prime}(Q) q_{i}<0
$$

guarantees the existence of equilibrium in a quantity-setting model since in this case the SOCs always hold. In the CC and the CV models, however, SOCs depend on $\alpha$ and $\lambda$ and (in a symmetric equilibrium) they are, respectively, given by

$$
\begin{gathered}
P^{\prime \prime}(Q) q_{i}(1+\alpha)+2 P^{\prime}(Q)-C^{\prime \prime}\left(q_{i}\right)<0 \\
(1+\lambda)\left(P^{\prime \prime}(Q) q_{i}(1+\lambda)+2 P^{\prime}(Q)\right)-C^{\prime \prime}\left(q_{i}\right)<0
\end{gathered}
$$

Then, since $C^{\prime \prime}\left(q_{i}\right) \geq 0,(1+\lambda) \geq 0$ and $(1+\alpha) \geq 0$, conditions [2] and [3] are satisfied if we assume $P^{\prime \prime}(Q) q_{i}(1+\alpha)+2 P^{\prime}(Q)<0$ and $P^{\prime \prime}(Q) q_{i}(1+\lambda)+2 P^{\prime}(Q)<0 .^{6}$ These assumptions certainly hold if demand is concave, but otherwise the degree of convexity must not be too large. The analysis of the $n$ symmetric FOCs leads us to the following result:

Proposition 1 The equilibrium quantities produced by firms in the CV model coincide with the ones in the CC model when $\lambda=\alpha$.

5 Admittedly $\alpha<0$ might seem counterintuitive at first sight. We follow here, however, the reasoning in Matsumura and Matsushima (2012) stating that objective functions are often based on relative performance since, for instance, evaluations of management activities are also based on their relative performances as well as the absolute performances. Furthermore, some experimental works already pointed out reciprocal or altruistic behavior (see, for instance, Cason, Saijo, and Yamato 2002 and Brandts et al. 2004) that is closely related to firms' objective functions based on relative performance (leading to either positive or negative $\alpha$ ).

6 We note that condition [3] coincides with condition [33] in Dixit (1986) for the case of constant conjectures. Additionally, we also note that if $\lambda=-1$, costs should be strictly convex in order for condition [3] to be satisfied. However, since the interest of the paper lies in the way to model imperfect competition, we will focus on the case where competition is not perfect. 
Proof. In the CV model, to maximize profits for firm $i$, we have to derive eq. [1] with respect to $q_{i}$ with $\left(\partial Q_{-i} / \partial q_{i}\right) \equiv \lambda$. Then, the FOC for the CV model for a generic firm $i$ becomes

$$
P(Q)+q_{i}[1+\lambda] \frac{\partial P\left(Q_{-i}+q_{i}\right)}{\partial q_{i}}=C^{\prime}\left(q_{i}\right)
$$

where $C^{\prime}\left(q_{i}\right)$ accounts for the marginal cost of production. On the other hand, in the CC model, the FOCs are obtained by maximizing

$$
\Pi_{i}\left(q_{i}, Q_{-i}\right)+\frac{\alpha}{n-1} \sum_{j \neq i}^{n} \Pi_{j}\left(q_{j}, Q_{-j}\right)
$$

with respect to $q_{i}$ leading to

$$
P(Q)+\frac{\partial P\left(Q_{-i}+q_{i}\right)}{\partial q_{i}} q_{i}+\frac{\alpha}{n-1}\left[\sum_{j \neq i}^{n} \frac{\partial P\left(Q_{-j}+q_{j}\right)}{\partial q_{i}} q_{j}\right]=C^{\prime}\left(q_{i}\right)
$$

In a symmetric equilibrium, $q_{i}=q_{j} \forall i \neq j$ and therefore

$$
\frac{\partial P\left(Q_{-j}+q_{j}\right)}{\partial q_{i}}=\frac{\partial P\left(Q_{-i}+q_{i}\right)}{\partial q_{i}}
$$

Hence, the FOC for firm $i$ becomes

$$
P(Q)+\frac{\partial P\left(Q_{-i}+q_{i}\right)}{\partial q_{i}} q_{i}+\frac{\alpha}{n-1}\left[\frac{\partial P\left(Q_{-i}+q_{i}\right)}{\partial q i} Q_{-i}\right]=C^{\prime}\left(q_{i}\right)
$$

Rearranging terms again we obtain

$$
P(Q)+\frac{\partial P\left(Q_{-i}+q_{i}\right)}{\partial q_{i}}\left[\frac{\alpha}{n-1} Q_{-i}+q_{i}\right]=C^{\prime}\left(q_{i}\right) .
$$

Recalling that symmetry implies that in equilibrium $Q=n q_{i}$ and $Q_{-i} \equiv Q-q_{i}$, the FOC for firm $i$ in the CC model can be written like

$$
P(Q)+q_{i} \frac{\partial P\left(Q_{-i}+q_{i}\right)}{\partial q_{i}}\left[1+(n-1) \frac{\alpha}{n-1}\right]=C^{\prime}\left(q_{i}\right)
$$

Thus,

$$
P(Q)+q_{i} \frac{\partial P\left(Q_{-i}+q_{i}\right)}{\partial q_{i}}[1+\alpha]=C^{\prime}\left(q_{i}\right)
$$

It is immediate to verify that the symmetric FOCs coincide in both models when $\lambda=\alpha$. Additionally, when $\lambda=\alpha$ both SOCs are satisfied simultaneously if for instance $P^{\prime \prime}(Q) q_{i}(1+\alpha)+2 P^{\prime}(Q)<0$. 
This result clearly indicates that the CV and the CC models are essentially equivalent when the conjectural variation expected and the coefficient of cooperation coincide. In other words, in a symmetric model of quantity-setting firms, the solution obtained when it is assumed that each firm expects a one-unit change in its quantity to lead to a change of $1+\lambda$ in total output replicates the solution obtained when each firm cares also about the (weighted by $\alpha$ ) average profits of the other firms. The strength of this result lies in the fact that for a wide range of symmetric oligopolies, we may estimate $\lambda$ and there always exists an equivalent value of the relative performance approach based on the coefficient of cooperation. This is important insofar as it legitimizes, for instance, comparative statics on an initial estimate of $\lambda$ (changing the oligopoly structure) based on changes in the reduced-form parameter $\alpha$ that, as pointed out by Matsumura et al. (2013), can be, for instance, also directly interpreted as a measure of altruism or the extent to which decision makers within firms care about relative performance. An additional interpretation of our analysis suggests that the nature of market competition might reside also in the well-known fact, pointed out by many behavioral economists, that reciprocity and non-self-interested motives affect people's decisions.

The present note, however, does not pretend to justify theoretically the possible flaws of the CV model where the conjectures assumed in the beginning might not be validated at the equilibrium. On the contrary, it has been shown here that perhaps theoretical and empirical results supported by the CV approach could easily be reinterpreted using a widely accepted approach that is consistent with the game-theoretic rationality and with the line of thought developed in the field of behavioral economics.

Acknowledgments: The author is grateful to Daniel Cardona, Noriaki Matsushima and an anonymous referee for helpful comments on an earlier draft of this paper. The usual disclaimer applies.

Funding: Financial support by the "Ministerio de Educación, Cultura y Deporte" through its project “Análisis de Economa Pública y Bienestar” (Ref: ECO201236480) is gratefully acknowledged.

\section{References}

Bowley, A. L. 1924. The Mathematical Groundwork of Economics. Oxford: Oxford University Press.

Brander, J. A., and A. Zhang 1990. "Market Conduct in the Airline Industry: An Empirical Investigation." The RAND Journal of Economics 21 (4):567-83. 
Brandts, J., T. Saijo, and A. Schram 2004. "How Universal Is Behavior? A Four Country Comparison of Spite and Cooperation in Voluntary Contribution Mechanisms" Public Choice 119 (3-4):381-424.

Bresnahan, T. F. 1981. "Duopoly Models with Consistent Conjectures.” The American Economic Review 71 (5):934-45.

Cabral, L. 1995. “Conjectural Variations as a Reduced Form.” Economics Letters 49 (4):397-402. Cason, T. N., T. Saijo, and T. Yamato 2002. "Voluntary Participation and Spite in Public Good Provision Experiments: An International Comparison." Experimental Economics 5 (2):133-53.

Charness, G., and M. Rabin 2002. "Understanding Social Preferences with Simple Tests." Quarterly Journal of Economics 17 (3):817-69.

Cyert, R. M., and M. H. DeGroot 1973. "An Analysis of Cooperation and Learning in a Duopoly Context." The American Economic Review 63 (1):24-37.

d'Aspremont, C., and R. Dos Santos Ferreira 2009. "Price-Quantity Competition with Varying Toughness." Games and Economic Behavior 65 (1):62-82.

Dixit, A. 1986. "Comparative Statics for Oligopoly." International Economic Review 27 (1): 107-22.

Dockner, E. J. 1992. “A Dynamic Theory of Conjectural Variations.” The Journal of Industrial Economics 40 (4):377-95.

Fehr, E., and K. M. Schmidt 1999. "A Theory of Fairness, Competition, and Cooperation." Quarterly Journal of Economics 114 (3):817-68.

Figuières, C., A. Jean-Marie, N. Quérou, and M. Tidball 2004. "Theory of Conjectural Variations." World Scientific (Series on Mathematical Economics and Game Theory) 2:33-64.

Kamien, M. I., and N. L. Schwartz 1983. "Conjectural Variations." Canadian Journal of Economics 16 (2):191-211.

Laitner, J. 1980. ““'Rational” Duopoly Equilibria.” The Quarterly Journal of Economics 95 (4):641-62.

Makowski, L. 1987. “Are 'Rational Conjectures' Rational?” Journal of Industrial Economics 36:35-47.

Martin, S. 2002. Advanced Industrial Economics 2nd ed. Oxford: Blackwell Publishers.

Matsumura, T., and N. Matsushima 2012. "Competitiveness and Stability of Collusive Behavior.” Bulletin of Economic Research 64 (1):22-31.

Matsumura, T., N. Matsushima, and S. Cato 2013. "Competitiveness and R\&D Competition Revisited." Economic Modelling 31:541-7.

Perry, M. K. 1982. "Oligopoly and Consistent Conjectural Variations." The Bell Journal of Economics 13 (1):197-205.

Slade, M. E. 1986. "Conjectures, Firm Characteristics, and Market Structure: An Empirical Assessment." International Journal of Industrial Organization 4 (4):347-69.

Symeonidis, G. 2000. "Price and Nonprice Competition with Endogenous Market Structure." Journal of Economics and Management Strategy 9 (1):53-83.

Symeonidis, G. 2008. "Downstream Competition, Bargaining, and Welfare.” Journal of Economics and Management Strategy 17 (1):247-70.

Tirole, J. 1988. The Theory of Industrial Organization. Cambridge: MIT Press.

Vega-Redondo, F. 1997. "The Evolution of Walrasian Behaviour." Econometrica 65 (2):375-84.

Vives, X. 2001. Oligopoly Pricing: Old Ideas and New Tools. Massachusetts: MIT Press. 